\section{El Bienestar Social asociado a los Valores Humanos en usuarios de transporte público de la Zona Sur del Gran Buenos Aires}

María Luciana Della Pittima

Facultad de Psicología y Relaciones Humanas, Universidad Abierta Interamericana, Argentina.

\section{Pablo Domingo Depaula}

Instituto de Investigaciones en Psicología, Facultad de Psicología, Universidad de Buenos Aires, Consejo Nacional de Investigaciones Científicas y Técnicas, Argentina.

Recibido: 8 de mayo de 2017. Aceptado: 10 de noviembre de 2017.

\section{Resumen}

El Bienestar Social es definido por Keyes (1998) como la valoración que hacemos de las circunstancias y el funcionamiento dentro de la sociedad. Los Valores Humanos son definidos como metas deseables que sirven a los intereses de alguna entidad social, que permiten adaptarse a la realidad de una sociedad y cultura (Schwartz, 2001). En la investigación realizada se han indagado las dimensiones del Bienestar Social y los Valores Humanos en usuarios de transporte público, con el objetivo de investigar si existen diferencias entre el contexto de los distintos medios de transporte y si se presenta una asociación entre dichas variables. A fines del año 2015 hasta mitad del siguiente, se seleccionó una muestra de 300 usuarios de transporte público, 149 pasajeros de colectivos y 151 usuarios de ferrocarril que realizan el recorrido de Zona Sur del Gran Buenos Aires a la Ciudad Autónoma de Buenos Aires. El estudio realizado fue de tipo cuantitativo, correlacional y de diferencia de grupos. Utilizando la Escala de Bienestar Social (Keyes, 1998) en su versión española traducida por Blanco y Díaz (2005) y la Escala de Valores (Schwartz et al., 2001). Los resultados mostraron que existen diferencias en las dimensiones del Bienestar Social, presentando una mejor percepción de las mismas aquellas personas que no utilizan el tren como medio de transporte.

\section{Palabras clave}

Bienestar social Valores humanos

Usuarios de transporte público Buenos Aires

\section{Palavras-chave}

Bem-Estar Social Valores humanos Usuários de transporte público Buenos Aires 


\section{The Social Well-being associated to the Human Values in users of public transport of the Southern Zone of Greater Buenos Aires}

\begin{abstract}
Social Well-being is defined by Keyes (1998) as the valuation we make of circumstances and functioning on the society. Human Values are defined as desirable goals that serve the interests of some social entity, allowing them to adapt to the reality of a society and culture (Schwartz, 2001). This research aimed to investigate the levels of Social Wellbeing and Human Values in users of public transport, if there are differences between the context of the transport and the relationship between this variables. By the end of 2015 until the middle of the following year, in order to verify these associations, a sample of 300 public transport users was selected, with 149 passengers of bus and 151 rail users doing the tour of Southern Zone of Greater Buenos Aires to Buenos Aires. The study was quantitative, correlational and differential groups. The instruments used to assess these variables were the Scale of Social Well-being (Keyes, 1998) in its Spanish version translated by Blanco and Díaz (2005) and Scale Values (Schwartz et al., 2001). The results showed that there are differences in the dimensions of Social Welfare, presenting a better perception of them people who do not use the train as a means of transportation.
\end{abstract}

\section{Introducción}

En los últimos años, en un contexto de profundo malestar en relación al servicio de transporte público de trenes de superficie, emergieron entre los usuarios distintas formas de expresar su disconformidad, sobrepasando los procedimientos institucionales en la resolución de los conflictos (Pérez, 2010).

Rebón et al. (2010), argumentan que en un contexto general de distintas formas de difusión y confrontación frente a las problemáticas y, en particular, de marcada disconformidad social con la forma de prestación del servicio de transporte ferroviario, surgieron una serie de hechos que requirieron la atención de los medios de comunicación masiva con diversas conceptualizaciones como "caos", "furia", "estallido" y "violencia". La investigación se llevó a cabo en el período en el que, como sostienen Pérez y Rebón (2017), para 2015 se alcanzó una mejora moderada que indicó la regularidad del servicio, aunque tales hechos no lograran representar evidencias conclusivas.

Al tratarse el servicio ferroviario de un sistema abierto, depende del intercambio con las condiciones de contorno, como las políticas, financiamiento y demanda social, con lo cual estos factores exógenos influyen de manera significativa en este sistema. A propósito de lo anterior, según Rebón y Hernández (2017:216), "las características propias que asume el sistema ferroviario lo constituyen en un espacio de una singular y múltiple conflictividad", existiendo un contexto dinámico subyacente al conflicto determinado por factores distinguibles; i.e., la actuación de los trabajadores sindicalizados y tercerizados a través de sus constantes reclamos, la calidad deficitaria del servicio que repercute sobre las mencionadas protestas incrementándolas, la disconformidad activa de los pasajeros debidas a las cancelaciones e interrupciones del servicio, sumado a las acciones de los familiares de las víctimas de accidentes ferroviarios, contextualizan el escenario actual de este importante sistema de transporte.

\section{Keywords}

Social Well-being Human Values Users of public transport Buenos Aires 
El desplazamiento para llevar a cabo diferentes actividades sociales, como trabajar, estudiar o tantos otros motivos, es una práctica social que implica la repetición de comportamientos sociales, en un contexto social, espacial y temporal especifica (Gutiérrez, 2009).

Para comprender las conductas tanto saludables como patológicas de las personas, es necesario que se las considere desde aspectos culturales, como los valores, creencias y expectativas, y desde los procesos psicológicos que aquellos moldean (Zubieta, Delfino y Fernández, 2007).

Frente a esta práctica que gran cantidad de personas realiza a diario, Pírez (1999) argumenta acerca de un sentimiento de desligamiento en cuanto a la responsabilidad social de cada ciudadano en el funcionamiento de la metrópoli, indicando una falta de la capacidad de decisión de aquellos en cuestiones sociales, teniendo en cuenta los servicios, en este caso los que se incluyen en el traslado de un lugar a otro. Con esto, podrían pensarse conductas en los usuarios de transporte púbico que para otras culturas o sociedades sean patológicas, como situaciones de estrés y malestar, llegando a generar agresividad, violencia y caos, pero que a partir de los aspectos culturales de esta sociedad, se hallan naturalizados.

Por otro lado, las investigaciones psicosociales acerca de la salud y el bienestar consideran las interacciones del individuo con el medio (Basabe, 2004). Se intenta incluir además de los aspectos psicológicos, los criterios sociales del bienestar, frente a la importancia de considerar tanto lo individual como lo social, lo que es dado y aquello que se construye en las interacciones con los demás y con el mundo.

Al hablar de Bienestar Social, se trasciende lo individual y la atención se centra en cómo se sienten los individuos dentro del medio en el que viven, si se consideran aptos y con la responsabilidad de tomar decisiones para las instituciones que cotidianamente habitan, si tienen una sensación de pertenencia a la sociedad, sus valores y creencias, si realmente se encuentran informados acerca de lo que ocurre realmente en el mundo en el que viven y, por otro lado, si se sienten ciudadanos con derechos y capacidad de aportar algo a la sociedad.

En suma, el Bienestar Social corresponde a la valoración que hacemos de las circunstancias y el funcionamiento dentro de la sociedad, destacándose la necesidad de considerar lo individual y lo social, el mundo dado y el mundo intersubjetivamente construido, ya que ambos aspectos de la existencia, tienen relación con el bienestar y con la salud (Keyes, 1998).

Bajo este panorama, el presente estudio pretende indagar la relación que existe entre los Valores Humanos y el Bienestar Social entre usuarios de tren de la Línea General Roca y usuarios de colectivos, apoyándose en la perspectiva de disciplinas diversas, que investigaron acerca del malestar creciente en los usuarios del ferrocarril.

Es de nuestra importancia la línea que conecta el Gran Buenos Aires (GBA) Sur, con la Ciudad Autónoma de Buenos Aires (CABA), específicamente el Ferrocarril General Roca (FCGR), ya que como indican los datos de la Comisión Nacional de Regulación del Transporte (CNRT; Ministerio de Transporte, 2015), es la línea que mayor cantidad de pasajeros transporta. En el sur del Área Metropolitana de Buenos Aires (AMBA), la empresa UGOFE (i.e., Unidad de Gestión Operativa Ferroviaria de Emergencia) cuenta con trenes de pasajeros de cinco ramales: Constitución - La Plata, Constitución - Cañuelas, Constitución - Alejandro Korn, Temperley - Haedo y Temperley - Bosques. 


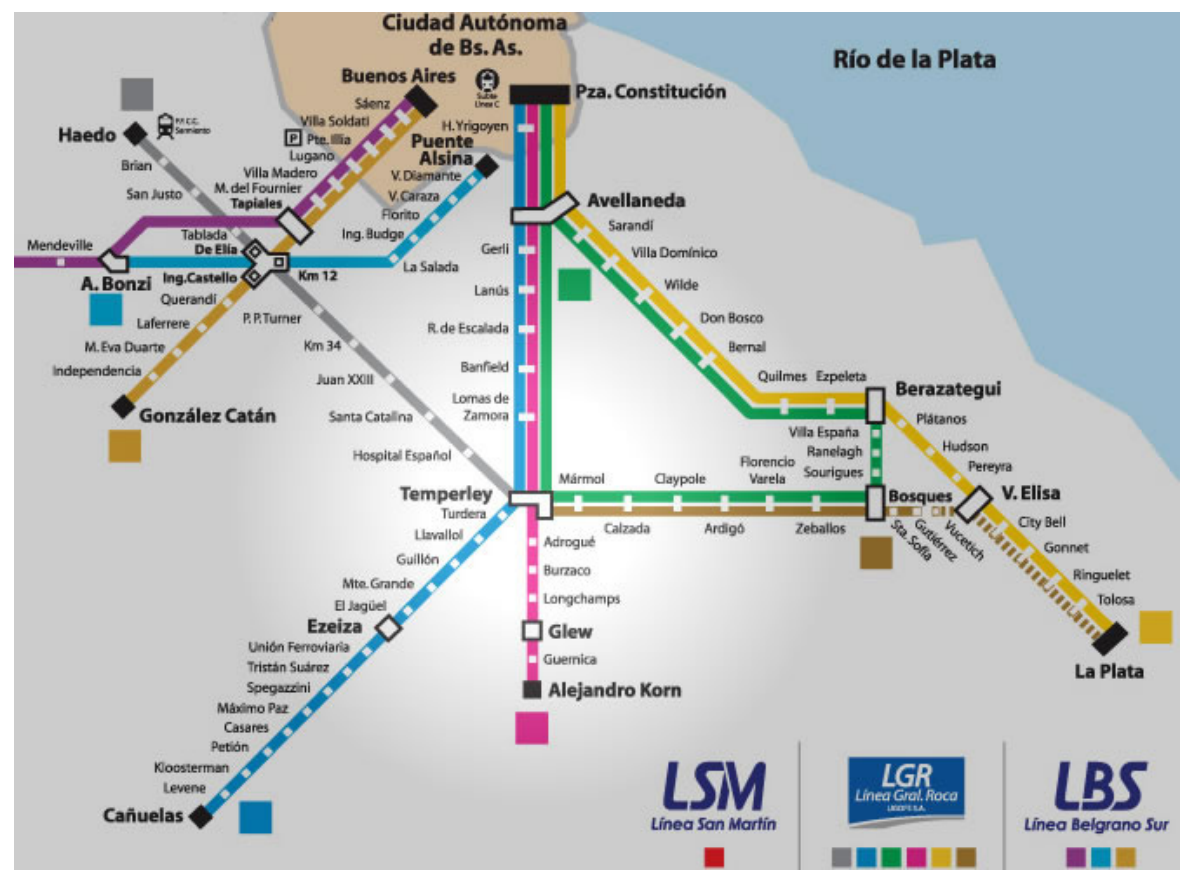

Gutiérrez (2009) argumenta acerca de lo que implica el "viaje o transporte" en una sociedad, considerándolo como una práctica social que involucra la repetición de comportamientos sociales, en un contexto social, espacial y temporal específico. Frente a esta práctica que gran cantidad de personas realiza a diario, como ya se ha adelantado arriba, Pírez (1999) enfatiza que existiría un desligamiento a nivel social de la responsabilidad de cada ciudadano en el funcionamiento de la urbe como una condición de la vida en grupo. Y continúa con la idea de que este hecho, indicaría una pérdida de la capacidad social de decisión sobre los servicios y de poder social sobre un componente básico de las condiciones de la vida en la ciudad. Este contexto de malestar frente a la desmejora del servicio de transporte, no cesa con el pasar de los años, haciéndose manifiesto en la caída de la calidad de vida de los usuarios del ferrocarril metropolitano de pasajeros de Buenos Aires (Müller, 2015).

\section{Sistema de transporte público en Argentina}

\section{Sistema de Transporte}

El Departamento de Transporte (2014 citado en Martínez et al., 2014:7), define al sistema de transporte como un "conjunto de instalaciones fijas (redes y terminales), entidades de flujo (vehículos) y un sistema de control que permiten movilizar eficientemente personas y bienes, para satisfacer necesidades humanas de movilidad".

A partir de la demanda que parte de las necesidades en cuanto al funcionamiento social, incluyendo la circulación de personas, bienes y servicios, acontece el surgimiento del transporte, por lo cual se denomina a éste como una "demanda derivada".

Las funciones básicas del sistema de transporte tienen que ver con la movilidad teniendo en cuenta la seguridad, comodidad, rapidez y economía en la circulación y el acceso que tienen las personas y vehículos a cualquier parte del país. Otro punto a tener en cuenta es la diferencia en cuanto a la demanda entre distintos sectores del país, momentos del año y horas del día, lo cual genera una dificultad en cuanto a las plataformas, si frente a la demanda cambiante se encuentran suficientemente preparadas (Martínez et al., 2014).
Figura 1. Mapa de recorridos de las líneas ferroviarias con destino a la Ciudad Autónoma de Buenos Aires. La "Línea General Roca” (LGR) con destino a la Estación “Plaza Constitución”, corresponde a la red ferroviaria analizada en el presente estudio. Fuente: extraído del sitio web http://www.temperleyweb.com.ar/galcdadest-mapa.jpg 


\section{Situación actual del transporte público de pasajeros}

Szenkman (2015) explica que en el último tiempo, la red de transporte del AMBA creció de forma exponencial, evidenciándose en datos que muestran desde el año 2003 al 2013, una suma de casi un millón de pasajeros por día. El automóvil particular, de ser poco utilizado, pasó a ser parte del $60 \%$ de los viajes, generando así un crecimiento del $80 \%$ de tráfico en los accesos a la CABA, lo cual influye inevitablemente en las demoras del transporte automotor de colectivos (sobre todo, en los accesos a la ciudad).

Sin embargo, el transporte colectivo creció más de un 30\%, generando un $80 \%$ de los pasajeros del transporte público metropolitano. En el caso de los ferrocarriles metropolitanos, se observó una evidente caída, cercana al $40 \%$ en la cantidad de pasajeros pagos transportados, pudiendo tener como causa la evasión en las líneas Mitre y Sarmiento, ya que no se cobró pasaje hasta tanto mejoraran las condiciones de prestación del servicio.

Entre el año 2003 hasta el año 2013, la demanda del transporte aumentó un 15\%, explicándose en el aumento del uso de colectivos, ya que el uso de los ferrocarriles bajó un $10 \%$.

Pérez y Rebón (2017) desarrollan que hubo un proceso de transformación en el servicio ferroviario en el período situado entre los años 2003 y 2015 considerando las situaciones problemáticas como disparadores para las mejoras de los servicios que devinieron. Incluyen en las condiciones del sistema ferroviario, factores exógenos que si bien trascienden el ámbito en el que se desarrolla la actividad, impactan sobre ella; entre ellos, la situación del país a principios del siglo XXI, la fuerza social con la que asumió el nuevo gobierno en ese momento y las condiciones económicas, sociales y políticas.

El ferrocarril forma parte de una alternativa importante al presentar altos niveles de accesibilidad económica, por los bajos costos de las tarifas, independientemente de la gran desmejora en los tiempos de viaje y el mal estado de la infraestructura. Según la Encuesta de Movilidad Domiciliaria (ENMODO; Presidencia de la Nación, 2009-2010), la preferencia por el modo Ferrocarril era mayor en las etapas de viaje originadas en el segundo cordón del GBA con destino a la CABA (siendo este uno de los modos preferidos para aquellos viajes dirigidos desde el hogar hacia el lugar de trabajo), reflejándose también la autonomía del Conurbano Bonaerense con respecto a la ciudad.

Según la mencionada encuesta, los hogares y las personas que realizaban más etapas de viaje utilizando el transporte público Ferrocarril pertenecían al primer y segundo quintil de ingresos per cápita de los hogares, i.e., los hogares que percibían ingresos más bajos, siendo entonces la restricción presupuestaria un factor que determinaba el motivo por el cual las personas podrían preferir este modo de transporte; asimismo, los encuestadores infirieron que las personas que se movilizaban bajo la modalidad Ferrocarril, arribaban a la estación a través del uso del colectivo o la bicicleta (en lugar de hacerlo caminando), siendo entonces el Ferrocarril solo una etapa de su viaje diario.

En la actualidad, se implementaron regulaciones para el control, mejoramiento y cierto seguimiento en el servicio de transporte público. Se llevó a cabo un Sistema Único de Boleto Electrónico, denominado "tarjeta SUBE", con el fin de generar beneficios como la ganancia de tiempo, comodidad, evitar falta de cambio y de monedas, y una mejora del medio ambiente por una menor utilización del papel. También, en el caso del transporte de colectivos, el Estado otorga subsidios para el gasoil, y se sancionó en el año 2013 la resolución 843/2013 que establece que todas las unidades cero kilómetro que se habiliten en el Servicio Público Urbano por Automotor de Jurisdicción Nacional, deberán estar equipadas con Aire Acondicionado, generando una mayor comodidad para los pasajeros (Martínez et al., 2014). 


\section{Acerca del concepto de bienestar social}

El bienestar social es definido por Keyes (1998) como la valoración que hacemos de las circunstancias y el funcionamiento dentro de la sociedad. Está compuesto por cinco dimensiones (Blanco \& Díaz, 2005; Keyes, 1998; Keyes \& Shapiro, 2004):

» Integración social: Tiene que ver con la evaluación de la calidad en las relaciones que mantenemos con la sociedad y con la comunidad. Las personas con alto bienestar social se sienten parte de la sociedad, cultivan el sentimiento de pertenencia y generan lazos sociales con la familia, amigos, vecinos y otros grupos sociales.

»Aceptación social: Refiere a la presencia de confianza, aceptación y actitudes positivas hacia los demás, como atribución de honestidad, bondad, amabilidad, capacidad y el reconocimiento de los aspectos positivos y negativos de nuestra propia vida.

»Contribución social: Es el sentimiento de ser útil, de que se es un miembro vital de la sociedad y que se tiene algo para ofrecer al mundo. Tiene que ver con el provecho, eficacia y aportación al bien común.

»Actualización social: La concepción de que la sociedad y las instituciones que la conforman son unidades eficientes, que se movilizan en una determinada dirección relacionada con la obtención de metas y objetivos de los que podemos beneficiarnos. Hay cierta confianza en el progreso y en el cambio social. Existe la seguridad en el futuro de la sociedad, en su capacidad de crecimiento y de desarrollo, y para producir bienestar.

» Coherencia social: Es la percepción de la cualidad, organización y funcionamiento del mundo social, e incluye el involucramiento por enterarse de lo que ocurre en el mundo. La sensación de que se es capaz de comprender lo que sucede en el contexto en el que vivimos.

\section{La Teoría de los Valores Humanos Básicos de Shalom Schwartz}

Schwartz (2001) propone una teoría universal sobre el significado y el contenido de los valores humanos. Los define como metas deseables, a través de situaciones, que varían en importancia y que sirven como principios rectores en la vida de una persona y para regular el funcionamiento social (Castro Solano, 2005).

Schwartz (2001) agrega que los valores sirven a los intereses de alguna entidad social, motivan las acciones, funcionan como criterios en los que se apoyan los juicios de valor y la justificación de algunos actos, siendo aprendidos y adquiridos a través de la socialización de los valores de un grupo dominante y mediante experiencias personales en el aprendizaje. Se tiene en cuenta que para poder adaptarse a la realidad de una sociedad y de determinada cultura, los diversos grupos y las personas transforman las necesidades propias de la existencia humana y las expresan en el lenguaje de los valores específicos a través de los cuales pueden comunicarse.

En un estudio que se desarrolló en más de 40 países, Schwartz (2001) encontró que había diez valores que aparecían en todas las culturas estudiadas, agrupándose todos ellos en dos dimensiones que se contrastan. El autor da relevancia al aspecto motivacional en la manera en que se ponen en juego estos valores, viéndose reflejado en cada uno metas y objetivos. Los mismos, se agrupan dependiendo del objetivo al que se pretenda llegar.

Por un lado, la autotrascendencia, dando prioridad a los intereses colectivos y lo grupal por sobre los intereses personales, se enfrenta a la autopromoción, en la que se priorizan los intereses propios. Por otro lado, la conservación, que se relaciona con las personas que aprecian la seguridad y el orden, se opone a la apertura al cambio, que predomina en aquellos sujetos que valoran la independencia de conductas y pensamientos. Estos pares 
que se oponen, delimitan los intereses sociales y culturales de cada persona. Pueden definirse intereses "colectivistas", aquellos valores que predominan en una sociedad y tienen que ver con atender a los intereses de la comunidad entera, e "individualistas", para los cuales se valoran aquellos aspectos que tienen que ver con el desarrollo y éxito personales, o bien "mixtos".

Páez y Zubieta (2006), elaboraron un cuestionario que incluían 56 valores obtenidos de las regiones más extendidas mundialmente y de estudios realizados en Asia y África. El estudio consistió en preguntar en qué medida cada uno de esos 56 valores eran representativos en cuanto a los principios que ordenaban sus vidas. Los autores mencionados sostienen que aunque los grupos e individuos varíen de manera significativa en el nivel de importancia que se les da a ciertos valores, se encuentran constituidos en cierta estructura general que establece las relaciones interpersonales.

Asimismo, Páez y Zubieta (2006) sosteniendo las ideas de Schwartz (2001), argumen$\tan$ que todas aquellas acciones que están orientadas a lograr un tipo de valor, tienen repercusiones psicológicas, prácticas y sociales que podrían no ser adecuadas para lograr la obtención de otros valores. Para Schwartz (2001), la prioridad de los valores puede manifestar la conducta social.

En consonancia con los aportes de Schwartz (2001), Castro Solano (2005) afirma que los valores representan las respuestas que las personas y los grupos deben dar a tres requisitos universales: (a) las necesidades de los individuos en su condición de seres biológicos, (b) la coordinación de las acciones sociales, y (c) el funcionamiento correcto y la supervivencia de los grupos.

Los diez tipos motivacionales de valores que se derivan de estos tres requisitos precisos son los siguientes:

» Poder: Interés por lograr poder social, autoridad, riqueza.

» Logro: Buscar el éxito personal poniendo en juego aptitudes que son socialmente aceptables.

» Hedonismo: Importan el placer y la gratificación personal, el poder disfrutar de la vida.

"Estimulación: Buscar la novedad y los desafíos.

» Autodirección: Interés por poder pensar de manera independiente, tener libertad de acción y exploración, poder ser creativo.

» Universalismo: Importancia a la tolerancia y la justicia social, la protección del medio ambiente.

" Benevolencia: Es importante preservar e intensificar el bienestar de las personas, la honestidad y la ausencia de rencor.

» Tradición: Interesa el respeto y el compromiso con las costumbres, y la cultura tradicional así como la religiosa.

»Conformidad: Se valoran las normas sociales y se evitan los comportamientos que puedan herir o molestar a los demás, se respeta a los mayores.

» Seguridad: Interesan la seguridad, armonía y estabilidad sociales y personales.

Al predominar algunos valores, pueden comprometer otros tipos de valores debido a las acciones que repercuten en aspectos psicológicos, prácticos y sociales; e.g., con el valor benevolencia, el logro puede entrar en conflicto, por lo tanto, las personas se acomodan a un modelo circular en el que los valores contiguos se relacionan. Por tal motivo, Schwartz (1992) relaciona logro y poder con la autopromoción y pone en contraposición a valores como el universalismo y la benevolencia, los cuales corresponden a la autotrascendencia. Generalmente, cuando aumenta el universalismo (que refiere a un aspecto social) disminuye la benevolencia (la cual se centra en un interés 
sobre las personas, pero de manera más individualista). El encuentro de estos valores, muestra que de manera continua se relacionan las motivaciones, formando un todo circular (Schwartz, 1992).

Fontaine, Poortinga, Delbeke y Schwartz (2008), proponen asociar los valores en dos grupos que se oponen; por un lado los que expresan los intereses personales, los cuales están centrados en la persona: autodirección, estimulación, hedonismo, logro y poder; y los que están focalizados en lo social, aquellos que tienen que ver con las relaciones interpersonales: universalismo, benevolencia, tradición, conformidad y seguridad.

Bilbao, Techio y Páez (2007), sostienen que las metas que se orientan a los valores basados en la autotrascendencia, son reforzadoras de las relaciones positivas que las personas tienen con quienes comparten su vida cotidiana.

Frente a las problemáticas que se presentan y reportan entre los usuarios del ferrocarril, es pertinente conocer los intereses de los usuarios de distintos transportes ya que inferimos que influyen a la hora de elegir el medio de desplazamiento, además en la percepción del modo en qué funciona la sociedad y las instituciones.

En suma, la presente investigación tuvo como objetivo general indagar si existía una relación entre las dimensiones del Bienestar Social y los Valores Humanos en los usuarios de diversos transportes públicos a partir de las diferencias que existen entre los valores que tienen que ver con la Autotrascendencia, los cuales están vinculados a intereses sociales mientras que aquellos que tienen que ver con la Autopromoción, contemplan intereses individualistas. Los objetivos específicos del estudio fueron los siguientes:

》 Indagar si exciten asociaciones entre el Bienestar Social y los Valores Humanos.

"Analizar diferencias de rangos del Bienestar Social y Valores Humanos entre usuarios de tren y de otros medios de transporte.

"Evaluar aspectos diferenciales del Bienestar Social en relación a variables sociodemográficas y características del modo de viaje (i.e., horario de ida y de vuelta, condiciones de comodidad de viaje, motivos de elcción del tipo de medio de transporte utilizado, y actividades realizadas usualmente durante el viaje).

\section{Metodología}

\section{Diseño}

Se realizó un estudio descriptivo correlacional y de diferencia de grupos -i.e., comparativo- (Hernández Sampieri, Fernández-Collado \& Baptista Lucio, 2006).

\section{Participantes}

Se seleccionó una muestra no probabilística por conveniencia integrada 300 usuarios: 151 usuarios de ferrocarril de la Línea General Roca, siendo la línea que mayor cantidad de pasajeros transportan, los que se trasladen diariamente de GBA Sur a Plaza Constitución (en los ramales Constitución - La Plata, Constitución - Cañuelas, Constitución - Alejandro Korn, Temperley - Haedo y Temperley - Bosques), y 149 usuarios de colectivos que se trasladen diariamente de GBA Sur a CABA contemplando el recorrido de las mismas zonas hacia CABA. La selección muestral y las características mencionadas se basaron en los indicadores recopilados por el Ministerio de Transporte a través de la CNRT (2015), ya mencionados en el apartado introductorio al presente estudio. 


\section{Instrumentos}

\section{Escala de Bienestar Social}

Para evaluar el Bienestar Social, se utilizó la Escala de Bienestar Social (Keyes, 1998) en su versión española traducida por Blanco y Díaz (2005), la cual evalúa la percepción de las personas sobre aspectos del entorno social que benefician el bienestar psicológico.

Está compuesta de 33 ítems respondibles a través de una escala tipo Likert, con continuo de respuesta de 1 (Totalmente en desacuerdo) a 5 (Totalmente de acuerdo); a través de la obtención de la puntuación media, mide la percepción que las personas tienen sobre cinco aspectos del entorno social que facilitan su bienestar psicológico: integración social $(\alpha=.71)$, aceptación social $(\alpha=.83)$, contribución social $(\alpha=.79)$, actualización social $(\alpha=.66)$ y coherencia social $(\alpha=.53)$.

En el estudio llevado a cabo por Keyes (1998), el coeficiente de fiabilidad para esta escala global fue muy satisfactorio $(\alpha=.85)$.

\section{Escala de Valores Humanos}

Para evaluar los valores humanos, se utilizó la Escala de Valores de Schwartz et al. (2001), en su versión resumida de 21 ítems. Los ítems describen características de las personas, evaluando las metas deseables que conducen al comportamiento social (por ejemplo: "Le parece importante tener ideas nuevas y ser creativo/a. Le gusta hacer las cosas de manera propia y original"). Las opciones de respuesta se presentan con una escala de tipo Likert, que van desde 0 (No se parece nada a mí) hasta 5 (Se parece a mí). La puntuación media de cada uno de los diez tipos motivacionales se obtiene calculando el cociente entre la sumatoria de los ítems correspondientes a cada tipo motivacional y el número de ítems de cada dimensión uno de estos. Sumando determinados valores, se forman cuatro grupos motivacionales contrastantes, a saber: apertura al cambio (autodirección, estimulación y hedonismo) vs. autopromoción (logro y poder); y autotrascendencia (universalismo y benevolencia) vs. conservación (tradición, conformidad y seguridad).

La fiabilidad de la escala original es aceptable en apertura al cambio $(\alpha=.65)$, en autopromoción ( $\alpha=.72)$, en auto-trascendencia $(\alpha=.65)$ y en conservación $(\alpha=.64)$.

\section{Cuestionario Socio-demográfico}

Plantea preguntas sociodemográficas relativas a: edad, sexo, estado civil, nivel educativo, zona de residencia, si tiene o no hijos, ocupación, remuneración mensual por su trabajo, horas de trabajo por día, la frecuencia con la que viaja a CABA, el motivo por el cual se traslada, si tiene algún vehículo propio y si lo tuviera, con qué frecuencia lo utilizaría para viajar a CABA.

\section{Cuestionario en relación al Uso del Servicio de Transporte Público}

Si realiza algún transbordo, el tiempo total de viaje, los medios de transporte que utiliza con más frecuencia, en qué horarios viaja de ida a CABA y vuelta a GBA Sur, con qué frecuencia viaja sentado, el motivo por el cual elije el medio de transporte que utiliza, que realiza durante el viaje, si viaja solo o acompañado y hace cuantos años viaja.

\section{Procedimiento}

Los datos ${ }^{1}$ fueron recolectados en el transporte de pasajeros "Ferrocarril Roca", en motivo de ser la línea que mayor cantidad de pasajeros transporta (como ya se ha
1. Teniendo en consideración los valores éticos de la investigación, se informó a cada persona encuestada el propósito general de la misma, la confidencialidad, que los datos serían anónimos y con fines académicos-científicos. 
mencionado arriba, según el Ministerio de Transporte, 2015); ramales Ezeiza, Alejandro Korn y Claypole, los cuales trasladan habitualmente pasajeros desde la zona sur del Gran Buenos Aires y a pasajeros de distintas líneas de transporte colectivos, i.e., las líneas 51, 74, 79, 45, 160 y 165 los cuales realizan recorridos desde diversos barrios de la Zona Sur de Gran Buenos Aires, coincidentes a estaciones del ferrocarril de la Línea Roca, hacia la CABA. Tanto los ramales de ferrocarril mencionados como las líneas 51 y 45, llegan al Centro de transbordo de Plaza Constitución, mientras que las líneas de ómnibus 74 y 79 llegan directamente al centro de la CABA. Las líneas 165 y 160 pasan por el centro de transbordo de Pompeya, y tienen como destino otras zonas de Capital Federal, como Once, Palermo y Ciudad Universitaria.

Sucesivamente, a los fines de incrementar el tamaño muestral sobre un número poblacional de tipo "desconocido" (pese a los datos estadísticos reportados acerca de la cantidad de pasajeros pagos por ramal durante el año 2015, e.g., 390.000 pasajeros pagos por día hábil; Ministerio de Transporte, 2015) se recolectaron datos a través de la red social Facebook (replicando los mismos cuestionarios, pero mediante una modalidad de administración de formato digital), en grupos de personas que utilizan estos medios de transporte. Frente a la predisposición de algunos encuestados de aplicar la misma batería de instrumentos a sujetos conocidos que cumplían con los criterios de inclusión, se utilizó en dicha instancia el muestreo de tipo "Bola de Nieve" el cual facilita que unos individuos conduzcan a otros, y éstos a terceros, llegando a obtener un tamaño muestral considerable (Hernández Sampieri et al., 2006), siendo estos casos incluidos en el total de los participantes encuestados $(N=300)$. Vale aclarar, que no se hallaron diferencias en las respuestas conforme al tipo de modalidad de administración utilizada para la encuesta (i.e., interceptación vs. bola de nieve).

La cantidad estimada de pasajeros pagos por día hábil comentada arriba, constituye un dato estadístico que relativiza en cierta forma la posibilidad de calcular con precisión el tamaño muestral debiendo considerar dicha cifra como un número poblacional "conocido", exacto; en tanto, e.g., los pasajeros "franquiciados" y los "evasores" del pago del boleto también formarían parte del fenómeno psicológico de estudio, con lo cual, definir un valor poblacional de $N$ pasajeros diarios "reales", resultaría impreciso. Considerado tales apreciaciones metodológicas, se procedió a llevar a cabo el cálculo del tamaño muestral "sin definir el número poblacional", asumiendo un nivel de confianza $Z_{c}$ para pruebas bilaterales de 1.96 , un valor de probabilidad de $p=.05$, un valor de no probabilidad de $q=.95 \mathrm{y}$, por último, un margen de error de $d=.05$, dando como resultado un tamaño muestral de no menos de 72 sujetos requeridos para que los datos recopilados de la encuesta sean confiables a un $95 \%$, i.e., que los resultados hallados alcancen un nivel de significancia de .05 .

\section{Resultados ${ }^{2}$}

Inicialmente, para verificar si las muestras respondían a una distribución normal, se realizó la prueba de normalidad a través del Test de Kolmogorov-Smirnov, mostrando una distribución asimétrica de la muestra; i.e., las variables Bienestar y Valores Humanos al Trabajo no respondían a una distribución normal $(p<.05)$. Por tanto, se realizaron análisis estadísticos no paramétricos: para el análisis de correlaciones se calculó el coeficiente Rho de Spearman, mientras que para diferenciar grupos se utilizó la prueba $U$ de Mann-Whittney (análisis de solo dos grupos).
2. Los datos fueron analizados mediante el Statistical Package for the Social Sciences (SPSS 21.o, versión en castellano; IBM Corporation, 1989, 2012). 


\section{Correlación entre Bienestar Social y Valores Humanos}

Primeramente, para analizar la relación entre las variables Bienestar Social y Valores Humanos se calculó el coeficiente Rho de Spearman, mostrando en el total de la muestra (independientemente del transporte modal utilizado) que los valores de Autotrascendencia, i.e., aquellos que tienen un carácter colectivo por sobre individual, se asociaron positiva y significativamente con todas las dimensiones del Bienestar Social; i.e., Universalismo: Integración ( $\rho=.145 ; p=.012)$, Aceptación ( $\rho=$ $.208 ; p=.000)$, Contribución $(\rho=.259 ; p=.000)$, Actualización $(\rho=.203 ; p=.000)$ y Coherencia $(\rho=.125 ; p=.030)$. Benevolencia: Integración $(\rho=.281 ; p=.000)$, Aceptación $(\rho=.227 ; p=.000)$, Contribución $(\rho=.214 ; p=.000)$, Actualización $(\rho=.264 ; p=.000)$ y Coherencia $(\rho=.212 ; p=.000)$ y Seguridad: Integración $(\rho=$ $.137 ; p=.018)$, Aceptación $(\rho=.217 ; p=.000)$, Contribución $(\rho=.157 ; p=.007)$, Actualización $(\rho=.171 ; p=.003)$ y Coherencia $(\rho=.118 ; p=.042)$. Dichos resultados podrían ser interpretados considerando que los Valores Humanos se caracterizan por su importancia hacia la tolerancia, el bienestar de las personas y la estabilidad social, prevaleciendo los intereses sociales por sobre los individuales; mientras que el Bienestar Social en general apunta a la valoración sobre el funcionamiento dentro de la sociedad, considerando aspectos como la confianza, aceptación, la seguridad social y el sentimiento de pertenencia.

Particularmente, en las dimensiones Integración Social, Actualización y Coherencia Social se halló una relación positiva y significativa con los valores de Apertura al cambio, Hedonismo, Estimulación y Autodirección, lo cual podría entenderse a partir de la posición activa de las personas en quienes predominan dichos valores hacia la búsqueda de la gratificación personal, un marcado interés por pensar de forma independiente y la búsqueda de desafíos, relacionándose entonces con las dimensiones del Bienestar Social mencionadas que tienen que ver con una concepción de la sociedad como una unidad eficiente, que va en busca de metas, sintiéndose los sujetos involucrados socialmente y con cierto nivel de entendimiento sobre el mundo. En el caso de la Aceptación Social asociada significativamente al valor Hedonismo $(\rho=.133 ; p=.022)$ y la Autodirección $(\rho=.146 ; p=.012)$, se destaca la confianza y las actitudes positivas hacia los demás en relación con el disfrute de la vida y el sentimiento de libertad de acción de quienes poseen un nivel más elevado de dichos valores. Por otro lado, la Contribución Social, considerada un sentimiento de utilidad y aportación social, posicionando al sujeto como un agente activo en la sociedad, se relaciona positiva y significativamente con los valores Estimulación $(\rho=.216 ; p=$ $.000)$, Autodirección $(\rho=.312 ; p=.000)$ y Logro $(\rho=.162 ; p=.005)$, los cuales tienen que ver con la búsqueda personal de éxito, desafíos y de libertad de acción.

El valor Conformismo se asocia positiva y significativamente con las dimensiones Integración Social $(\rho=.144 ; p=.013)$ y Aceptación Social $(\rho=.162 ; p=.005)$; dichas dimensiones se relacionan con la aceptación hacia los demás, un sentimiento de pertenencia, la presencia de confianza y actitudes positivas hacia los demás, relacionándose con el respeto hacia los demás y a las normas sociales que caracteriza al Conformismo.

El Valor Poder se asoció negativa y significativa con las dimensiones Aceptación ( $\rho=$ $-.124 ; p=.032)$, Contribución $(\rho=-.137 ; p=.018)$ y Coherencia Social $(\rho=-.158 ; p=$ .006), i.e., cuanto más alta es la puntuación en estas dimensiones, más bajo es el nivel en el Poder. Las mencionadas dimensiones del Bienestar Social apuntan a intereses sociales de aceptación hacia los demás, el sentimiento de pertenencia y las actitudes positivas hacia los demás, mientras el valor Poder, posee un interés de autoridad, poniendo énfasis en lo individual. 
Cuadro 1. Correlación entre Bienestar Social y Valores. Fuente: elaboración propia en base al reporte de resultados del paquete estadístico SPSS 21.0. ${ }^{* *}$. La correlación es significativa al nivel 0.01 (bilateral). *. La correlación es significativa al nivel o.05 (bilateral).

\begin{tabular}{|c|c|c|c|c|c|}
\hline & Integración Social & Aceptación Social & $\begin{array}{l}\text { Contribución } \\
\text { Social }\end{array}$ & $\begin{array}{l}\text { Actualización } \\
\text { Social }\end{array}$ & Coherencia Social \\
\hline Autotrascendencia & $\begin{array}{c}\text { Rho }=.228 * * \\
\text { Sig. }=.000\end{array}$ & $\begin{array}{l}.276 * * \\
.000\end{array}$ & $\begin{array}{l}.269^{* * *} \\
.000\end{array}$ & $\begin{array}{l}.281^{* * *} \\
.000\end{array}$ & $\begin{array}{l}.182^{* * *} \\
.000\end{array}$ \\
\hline Universalismo & $\begin{array}{c}\text { Rho }=.145^{*} \\
\text { Sig. }=.012\end{array}$ & $\begin{array}{l}.208 * * \\
.000\end{array}$ & $\begin{array}{l}.259 * * \\
.000\end{array}$ & $\begin{array}{l}.203^{* *} \\
.000\end{array}$ & $\begin{array}{l}.125^{*} \\
.030\end{array}$ \\
\hline Benevolencia & $\begin{array}{c}\text { Rho }=.281^{* *} \\
\text { Sig. }=.000\end{array}$ & $\begin{array}{l}.227^{* * *} \\
.000\end{array}$ & $\begin{array}{l}.214^{* * *} \\
.000\end{array}$ & $\begin{array}{l}.264^{* * *} \\
.000\end{array}$ & $\begin{array}{l}.212^{* * *} \\
.000\end{array}$ \\
\hline Conservación & $\begin{array}{c}\text { Rho }=.175^{* * *} \\
\text { Sig. }=.003\end{array}$ & $\begin{array}{l}.160 \% * \\
.006\end{array}$ & $\begin{array}{l}.139^{*} \\
.016\end{array}$ & $\begin{array}{l}.157^{* * *} \\
.007\end{array}$ & $\begin{array}{l}.038 \\
.512\end{array}$ \\
\hline Tradición & $\begin{array}{c}\text { Rho }=-.025 \\
\text { Sig. }=.674\end{array}$ & $\begin{array}{l}-.087 \\
.132\end{array}$ & $\begin{array}{l}-.059 \\
.306\end{array}$ & $\begin{array}{l}.001 \\
.991\end{array}$ & $\begin{array}{l}-.067 \\
.245\end{array}$ \\
\hline Seguridad & $\begin{array}{l}\text { Rho }=.137^{*} \\
\text { Sig. }=.018\end{array}$ & $\begin{array}{l}.217^{* * *} \\
.000\end{array}$ & $\begin{array}{l}.157^{* * *} \\
.007\end{array}$ & $\begin{array}{l}.171^{* * *} \\
.003\end{array}$ & $\begin{array}{l}.118^{*} \\
.042\end{array}$ \\
\hline Conformismo & $\begin{array}{c}\text { Rho }=.144^{*} \\
\text { Sig. }=.013\end{array}$ & $\begin{array}{l}.162^{* * *} \\
.005\end{array}$ & $\begin{array}{l}.106 \\
.067\end{array}$ & $\begin{array}{l}.085 \\
.144\end{array}$ & $\begin{array}{l}-.013 \\
.821\end{array}$ \\
\hline Autopromoción & $\begin{array}{l}\text { Rho }=.037 \\
\text { Sig. }=.529\end{array}$ & $\begin{array}{l}-.001 \\
.983\end{array}$ & $\begin{array}{l}.037 \\
.525\end{array}$ & $\begin{array}{l}-.065 \\
.266\end{array}$ & $\begin{array}{l}-.047 \\
.421\end{array}$ \\
\hline Poder & $\begin{array}{c}\text { Rho }=-.016 \\
\text { Sig. }=.787\end{array}$ & $\begin{array}{l}-.124 * \\
.032\end{array}$ & $\begin{array}{l}-.137^{*} \\
.018\end{array}$ & $\begin{array}{l}.212^{* * *} \\
.000\end{array}$ & $\begin{array}{l}-.158 * * \\
.006\end{array}$ \\
\hline Logro & $\begin{array}{c}\text { Rho }=.091 \\
\text { Sig. }=.118\end{array}$ & $\begin{array}{l}.094 \\
.104\end{array}$ & $\begin{array}{l}.162^{* * *} \\
.005\end{array}$ & $\begin{array}{l}.065 \\
.265\end{array}$ & $\begin{array}{l}.067 \\
.250\end{array}$ \\
\hline Apertura al cambio & $\begin{array}{c}\text { Rho }=.269^{* * *} \\
\text { Sig. }=.000\end{array}$ & $\begin{array}{l}.164^{* * *} \\
.004\end{array}$ & $\begin{array}{l}.226 * * \\
.000\end{array}$ & $\begin{array}{l}.274^{* * *} \\
.000\end{array}$ & $\begin{array}{l}.239 * * \\
.000\end{array}$ \\
\hline Hedonismo & $\begin{array}{c}\text { Rho }=.141^{*} \\
\text { Sig. }=.015\end{array}$ & $\begin{array}{l}.133^{*} \\
.022\end{array}$ & $\begin{array}{l}.097 \\
.093\end{array}$ & $\begin{array}{l}.200 * * * \\
.001\end{array}$ & $\begin{array}{l}.154^{* * *} \\
.007\end{array}$ \\
\hline Estimulación & $\begin{aligned} \text { Rho } & =.251^{* * *} \\
\text { Sig. } & =.000\end{aligned}$ & $\begin{array}{l}.107 \\
.063\end{array}$ & $\begin{array}{l}.216^{* *} \\
.000\end{array}$ & $\begin{array}{l}.242^{* * *} \\
.000\end{array}$ & $\begin{array}{l}.208 * * \\
.000\end{array}$ \\
\hline Autodirección & $\begin{array}{c}\text { Rho }=.283^{* * *} \\
\text { Sig. }=.000\end{array}$ & $\begin{array}{l}.146^{*} \\
.012\end{array}$ & $\begin{array}{l}.312^{* * *} \\
.000\end{array}$ & $\begin{array}{l}.259^{* * *} \\
.000\end{array}$ & $\begin{array}{l}.248 * * \\
.000\end{array}$ \\
\hline
\end{tabular}

Diferencias de rangos del Bienestar Social y Valores Humanos entre usuarios de tren y de otros medios de transporte

$\mathrm{Al}$ diferenciar los rangos entre los usuarios de tren y otros medios de transporte se observaron diferencias significativas en todas las dimensiones del Bienestar Social, interpretando de esta manera que aquellas personas que utilizan el tren como medio de preferencia para movilizarse, tienen baja puntuación en dichas dimensiones, i.e., tienen una valoración negativa del funcionamiento de la sociedad, mientras que aquellos que viajan en otro medio de transporte obtuvieron elevados niveles, considerando que poseen una percepción positiva con respecto a la valoración y funcionamiento de la sociedad. Los resultados dimensionales de la variable Bienestar Social relativos al análisis estadístico anterior son los siguientes: Integración ( $U$ de Mann-Whittney = 6441,500; $p=.000$ ), Aceptación ( $U$ de Mann-Whittney $=5283,500 ; p=.000$ ), Contribución $(U$ de Mann-Whittney $=6152,500 ; p=.000)$, Actualización ( $U$ de Mann-Whittney $=$ 6810,$000 ; p=.000$ ) y Coherencia ( $U$ de Mann-Whittney $=7309,500 ; p=.000$ ).

$\mathrm{Al}$ analizar las diferencias entre los usuarios de ferrocarril y aquellos que no lo utilizan, se evidenciaron mayores puntuaciones en aquellos que no utilizan el tren como medio de transporte preferente en los Valores Humanos, Benevolencia ( $U$ de Mann-Whittney = 8090,$000 ; p=.000$ ), Conformismo ( $U$ de Mann-Whittney $=7705,000 ; p=.000$ ), Seguridad ( $U$ de Mann-Whittney $=7302,000 ; p=.000)$ : los cuales refieren a la importancia 
de las relaciones cercanas y la armonía con los demás; Logro ( $U$ de Mann-Whittney = $8577,000 ; p=.000$ ) y Hedonismo ( $U$ de Mann-Whittney = 8881,000; $p=.001)$ : dando importancia a la satisfacción personal e individual; Estimulación ( $U$ de Mann-Whitt$n e y=9505,500 ; p=.019)$ y Autodirección ( $U$ de Mann-Whittney $=9086,000 ; p=.006$ ); predominando el interés por lo novedoso y la libertad de acción.

Cuadro 2. Diferencias de rangos del Bienestar Social y Valores Humanos entre usuarios de tren y de otros medios de transporte. Fuente: elaboración propia en base al reporte de resultados del paquete estadístico SPSS 21.0.

\begin{tabular}{|l|c|c|c|c|c|}
\hline & $\begin{array}{c}\text { Rango Promedio } \\
\text { Usuarios de tren }\end{array}$ & $\begin{array}{c}\text { Rango Promedio } \\
\text { No viajan en tren }\end{array}$ & $\begin{array}{c}\text { U de Mann- } \\
\text { Whittney }\end{array}$ & Z & Sig. \\
\hline Integración Social & 118,44 & 180,18 & 6441,500 & $-6,228$ & .000 \\
Aceptación Social & 110,99 & 190,54 & 5283,500 & $-7,956$ & .000 \\
Contribución Social & 116,75 & 184,71 & 6152,500 & $-6,815$ & .000 \\
Actualización Social & 121,10 & 178,67 & 6810,000 & $-5,795$ & .000 \\
Coherencia Social & 124,41 & 176,94 & 7309,500 & $-5,269$ & .000 \\
\hline Autotrascendencia & 133,17 & 168,06 & 8633,000 & $-3,488$ & .000 \\
\hline Universalismo & 141,82 & 159,29 & 9939,500 & $-1,762$ & .078 \\
\hline Benevolencia & 129,58 & 171,70 & 8090,000 & $-4,320$ & .000 \\
\hline Conservación & 125,88 & 174,28 & 7557,000 & $-4,854$ & .000 \\
\hline Tradición & 151,18 & 148,82 & 10998,500 &,- 239 & .811 \\
\hline Seguridad & 124,36 & 176,99 & 7302,000 & $-5,310$ & .000 \\
\hline Conformismo & 127,03 & 174,29 & 7705,000 & $-4,754$ & .000 \\
\hline Autopromoción & 138,16 & 163,01 & 9385,500 & $-2,491$ & .013 \\
\hline Poder & 147,14 & 153,90 & 10742,500 &,- 684 & .494 \\
\hline Logro & 132,80 & 169,44 & 8577,000 & $-3,592$ & .000 \\
\hline Apertura al cambio & 134,15 & 164,85 & 8814,000 & $-3,081$ & .002 \\
\hline Hedonismo & 134,81 & 166,40 & 8881,000 & $-3,191$ & .001 \\
\hline Estimulación & 138,95 & 162,20 & 9505,500 & $-2,341$ & .019 \\
\hline Autodirección & 135,98 & 163,02 & 9086,000 & $-2,758$ & .006 \\
\hline
\end{tabular}

\section{Aspectos diferenciales del Bienestar Social en relación a variables sociodemográficas y características del modo de viaje}

Acto seguido, se decidió analizar diferencias en los niveles de Bienestar Social conforme a una variable o atributo de la práctica del viaje (más que a cualidades de los sujetos encuestados) que caracteriza al modo de transporte elegido y posee relevancia para el presente estudio, i.e., el "horario" en que las personas utilizan los medios de transporte en cuestión, dado que existirían aspectos tales como la cantidad de pasajeros que utilizan el mismo medio con mayor frecuencia en una franja horaria determinada por cuestiones laborales (i.e., "horas pico"), medidas diurnas de seguridad, etc. Las "horas pico" se caracterizan por la gran cantidad de personas que se trasladan en el mismo momento, generando hacinamientos y demoras en los traslados, mientras que quienes viajan a contra tiempo, tienen otras condiciones de viaje por ejemplo con menor cantidad de pasajeros.

En relación a la variable de horario de viaje de Ida a CABA se encontraron diferencias significativas en tres dimensiones del Bienestar Social; Integración Social $\left(X^{2}=8.598\right.$, $g l=2, p=.014)$, Aceptación Social $\left(X^{2}=31.745, g l=2, p=.000\right)$ y Actualización Social $\left(X^{2}=16.745, g l=2, p=.000\right)$, observándose que aquellos que viajan "después de las 09:00 am" poseen niveles más elevados en estas dimensiones que aquellos que viajan previo a dicho horario. 
Cuadro 3. Bienestar Social y horario de viaje de ida. Notas: a. Prueba de Kruskal-Wallis; b. Variable de agrupación: Horario de viaje: Ida a CABA. Fuente: elaboración propia en base al reporte de resultados del paquete estadístico SPSS 21.0.

\begin{tabular}{|c|c|c|c|}
\hline \multicolumn{3}{|c|}{ Horario de viaje: Ida a CABA: } & Rango promedio \\
\hline \multirow[t]{3}{*}{ Integración Social } & \multicolumn{2}{|c|}{ antes de las 07:00 am } & 139.54 \\
\hline & \multicolumn{2}{|c|}{ de 07:00 a 09:00 am } & 141.02 \\
\hline & \multicolumn{2}{|c|}{ después de las 09:00 am } & 173.98 \\
\hline \multirow[t]{3}{*}{ Aceptación Social } & \multicolumn{2}{|c|}{ antes de las 07:00 am } & 148.62 \\
\hline & \multicolumn{2}{|c|}{ de 07:00 a 09:00 am } & 128.71 \\
\hline & \multicolumn{2}{|c|}{ después de las 09:00 am } & 197.29 \\
\hline \multirow[t]{3}{*}{ Actualización Social } & \multicolumn{2}{|c|}{ antes de las 07:00 am } & 149.63 \\
\hline & \multicolumn{2}{|c|}{ de 07:00 a 09:00 am } & 133.28 \\
\hline & \multicolumn{2}{|c|}{ después de las 09:00 am } & 182.69 \\
\hline \multicolumn{4}{|c|}{ Estadísticos de contraste } \\
\hline & Integración Social & Aceptación Social & Actualización Social \\
\hline Chi-cuadrado & 8.598 & 31.745 & 16.745 \\
\hline $\mathrm{Gl}$ & 2 & 2 & 2 \\
\hline Sig. asintót. & .014 & .000 & .000 \\
\hline
\end{tabular}

Lo mismo ocurre en el horario de vuelta a GBA Sur, presentando mayores niveles en todas las dimensiones del Bienestar Social aquellos usuarios que viajan "antes de las 17:00 hs."; Integración Social $\left(X^{2}=19.822, g l=2, p=.000\right)$, Aceptación Social $\left(X^{2}=\right.$ $13.011, g l=2, p=.001)$, Contribución Social $\left(X^{2}=13.720, g l=2, p=.001\right)$, Actualización Social $\left(X^{2}=10.961, g l=2, p=.004\right)$ y Coherencia Social $\left(X^{2}=16.652, g l=2, p=.000\right)$.

Cuadro 4. Bienestar Social y horario de viaje de vuelta. Notas: a. Prueba de KruskalWallis; b. Variable de agrupación: Horario de viaje: Vuelta a GBASur. Fuente: elaboración propia en base al reporte de resultados del paquete estadístico SPSS 21.0.

\begin{tabular}{|l|c|c|}
\hline \multirow{4}{*}{ Integración Social } & Horario de viaje: Vuelta a GBA & Rango promedio \\
\cline { 2 - 3 } & antes de las 17:00 hs. & 175.74 \\
\cline { 2 - 3 } & de 17:00 a 19:00 hs. & 127.94 \\
\hline Aceptación Social & antes de las 17:00 hs. & 159.30 \\
\cline { 2 - 3 } & de 17:00 a 19:00 hs. & 172.83 \\
\cline { 2 - 3 } & después de las 19:00 hs. & 133.43 \\
\hline Contribución Social & antes de las 17:00 hs. & 155.78 \\
\cline { 2 - 3 } & de 17:00 a 19:00 hs. & 175.50 \\
\cline { 2 - 3 } & después de las 19:00 hs. & 138.49 \\
\hline Actualización Social & antes de las 17:00 hs. & 132.07 \\
\cline { 2 - 3 } & de 17:00 a 19:00 hs. & 164.27 \\
\cline { 2 - 3 } & después de las 19:00 hs & 133.38 \\
\hline Coherencia Social & antes de las 17:00 hs & 170.23 \\
\cline { 2 - 3 } & de 17:00 a 19:00 hs & 178.11 \\
\cline { 2 - 3 } & después de las 19:00 hs & 134.44 \\
\hline
\end{tabular}




\begin{tabular}{|l|c|c|c|c|c|}
\hline \multicolumn{7}{|c|}{ Estadísticos de contraste $^{\mathrm{a}, \mathrm{b}}$} \\
\hline & $\begin{array}{c}\text { Integración } \\
\text { Social }\end{array}$ & $\begin{array}{c}\text { Aceptación } \\
\text { Social }\end{array}$ & $\begin{array}{c}\text { Contribución } \\
\text { Social }\end{array}$ & $\begin{array}{c}\text { Actualización } \\
\text { Social }\end{array}$ & $\begin{array}{c}\text { Coherencia } \\
\text { Social }\end{array}$ \\
\hline Chi-cuadrado & 19.822 & 13.011 & 13.720 & 10.961 & 16.652 \\
\hline Gl & 2 & 2 & 2 & 2 & 2 \\
\hline Sig. asintót. & .000 & .001 & .001 & .004 & .000 \\
\hline
\end{tabular}

Asimismo, en todas las dimensiones del Bienestar Social, se hallaron diferencias significativas en cuanto a la "manera y a la regularidad en la que viajan" los pasajeros. Se observaron niveles más altos de Bienestar Social en aquellos sujetos que respondieron que "a veces", "frecuentemente" o "siempre" viajan sentados.

Cuadro 5. Bienestar Social y condición de comodidad de viaje. Notas: a. Prueba de Kruskal-Wallis; b. Variable de agrupación: ¿Viaja sentado?. Fuente: elaboración propia en base al reporte de resultados del paquete estadístico SPSS 21.0.

\begin{tabular}{|c|c|c|c|c|c|}
\hline & & \multicolumn{2}{|c|}{ ¿Viaja sentado? } & \multicolumn{2}{|c|}{ Rango promedio } \\
\hline \multirow{5}{*}{\multicolumn{2}{|c|}{ Integración Social }} & \multicolumn{2}{|c|}{ SIEMPRE } & \multicolumn{2}{|c|}{164.20} \\
\hline & & \multicolumn{2}{|c|}{ FRECUENTEMENTE } & \multicolumn{2}{|c|}{185.52} \\
\hline & & \multicolumn{2}{|c|}{ A VECES } & \multicolumn{2}{|c|}{170.73} \\
\hline & & \multicolumn{2}{|c|}{ RARA VEZ } & \multicolumn{2}{|c|}{114.41} \\
\hline & & \multicolumn{2}{|c|}{ NUNCA } & \multicolumn{2}{|c|}{109.36} \\
\hline \multirow{5}{*}{\multicolumn{2}{|c|}{ Aceptación Social }} & \multicolumn{2}{|c|}{ SIEMPRE } & \multicolumn{2}{|c|}{175.55} \\
\hline & & \multicolumn{2}{|c|}{ FRECUENTEMENTE } & \multicolumn{2}{|c|}{190.24} \\
\hline & & \multicolumn{2}{|c|}{ A VECES } & \multicolumn{2}{|c|}{175.31} \\
\hline & & \multicolumn{2}{|c|}{ RARA VEZ } & \multicolumn{2}{|c|}{129.03} \\
\hline & & \multicolumn{2}{|c|}{ NUNCA } & \multicolumn{2}{|c|}{84.36} \\
\hline \multirow{5}{*}{\multicolumn{2}{|c|}{ Contribución Social }} & \multicolumn{2}{|c|}{ SIEMPRE } & \multicolumn{2}{|c|}{175.19} \\
\hline & & \multicolumn{2}{|c|}{ FRECUENTEMENTE } & \multicolumn{2}{|c|}{183.74} \\
\hline & & \multicolumn{2}{|c|}{ A VECES } & \multicolumn{2}{|c|}{165.68} \\
\hline & & RAR & VEZ & & \\
\hline & & $\mathrm{NU}$ & CA & & \\
\hline Actualización Socia & & SIEN & PRE & & \\
\hline & & FRECUEN & EMENTE & & \\
\hline & & $\mathrm{A} V \mathrm{~V}$ & CES & & \\
\hline & & RAR & VEZ & & \\
\hline & & $\mathrm{NU}$ & CA & & \\
\hline Coherencia Social & & SIEN & PRE & & \\
\hline & & FRECUEN & EMENTE & & \\
\hline & & $\mathrm{AVV}$ & CES & & \\
\hline & & RAR & VEZ & & \\
\hline & & NU & CA & & \\
\hline & & tadísticos de & ontraste ${ }^{a, b}$ & & \\
\hline & $\begin{array}{l}\text { Integración } \\
\text { Social }\end{array}$ & $\begin{array}{l}\text { Aceptación } \\
\text { Social }\end{array}$ & $\begin{array}{l}\text { Contribución } \\
\text { Social }\end{array}$ & & $\begin{array}{c}\text { Coherencia } \\
\text { Social }\end{array}$ \\
\hline Chi-cuadrado & 41.014 & 60.868 & 40.470 & 42.412 & 41.384 \\
\hline $\mathrm{Gl}$ & 4 & 4 & 4 & 4 & 4 \\
\hline Sig. asintót. & .000 & .000 & .000 & .000 & .000 \\
\hline
\end{tabular}


Se observa que ocurre lo mismo en relación al "motivo por el cual eligen el medio de transporte"; los sujetos que lo hacen por "comodidad", presentan niveles superiores de Bienestar Social que aquellos que optan por otros motivos, como la "rapidez".

Cuadro 6. Bienestar Social y motivo de elección del transporte. Notas: a. Prueba de Kruskal-Wallis; $b$. Variable de agrupación: ¿Por qué motivo elige los medios de transporte que utiliza más frecuentemente?. Fuente: elaboración propia en base al reporte de resultados del paquete estadístico SPSS 21.0.

\begin{tabular}{|c|c|c|}
\hline & $\begin{array}{l}\text { ¿Por qué motivo elige los medios de } \\
\text { transporte que utiliza más frecuentemente? }\end{array}$ & Rango promedio \\
\hline \multirow[t]{5}{*}{ Integración Social } & RAPIDEZ & 130.63 \\
\hline & ECONOMICO & 129.20 \\
\hline & CERCANIA A PARADA O ESTACION & 149.91 \\
\hline & COMODIDAD & 177.06 \\
\hline & SEGURIDAD & 213.25 \\
\hline \multirow[t]{5}{*}{ Aceptación Social } & RAPIDEZ & 119.87 \\
\hline & ECONOMICO & 117.89 \\
\hline & CERCANIA A PARADA O ESTACION & 138.43 \\
\hline & COMODIDAD & 205.18 \\
\hline & SEGURIDAD & 27.00 \\
\hline \multirow[t]{5}{*}{ Contribución Social } & RAPIDEZ & 121.92 \\
\hline & ECONOMICO & 132.32 \\
\hline & CERCANIA A PARADA O ESTACION & 158.69 \\
\hline & COMODIDAD & 191.20 \\
\hline & SEGURIDAD & 57.00 \\
\hline \multirow[t]{5}{*}{ Actualización Social } & RAPIDEZ & 120.97 \\
\hline & ECONOMICO & 149.33 \\
\hline & CERCANIA A PARADA O ESTACION & 146.98 \\
\hline & COMODIDAD & 186.98 \\
\hline & SEGURIDAD & 26.00 \\
\hline \multirow[t]{5}{*}{ Coherencia Social } & RAPIDEZ & 122.39 \\
\hline & ECONOMICO & 124.37 \\
\hline & CERCANIA A PARADA O ESTACION & 136.93 \\
\hline & COMODIDAD & 197.69 \\
\hline & SEGURIDAD & 148.00 \\
\hline
\end{tabular}

\begin{tabular}{|l|c|c|c|c|c|}
\hline \multicolumn{7}{|c|}{ Estadísticos de contraste } \\
\hline & $\begin{array}{c}\text { Integración } \\
\text { Social }\end{array}$ & $\begin{array}{c}\text { Aceptación } \\
\text { Social }\end{array}$ & $\begin{array}{c}\text { Contribución } \\
\text { Social }\end{array}$ & $\begin{array}{c}\text { Actualización } \\
\text { Social }\end{array}$ & $\begin{array}{c}\text { Coherencia } \\
\text { Social }\end{array}$ \\
\hline Chi-cuadrado & 20.252 & 67.933 & 41.341 & 37.575 & 49.031 \\
\hline Gl & 4 & 4 & 4 & 4 & 4 \\
\hline Sig. asintót. & .000 & .000 & .000 & .000 & .000 \\
\hline
\end{tabular}

Acto seguido, se observó que aquellos sujetos que utilizan el tren como medio de preferencia, lo hacen por rapidez, mientras que una parte importante de la muestra que elige como medio de transporte otro diferente al ferrocarril, lo hacen por comodidad. 
Cuadro 7. Motivo de elección del transporte en usuarios de tren y quienes no viajan en tren. Fuente: elaboración propia en base al reporte de resultados del paquete estadístico SPSS 21.0.

\begin{tabular}{|c|c|c|c|c|}
\hline \multirow{2}{*}{\multicolumn{2}{|c|}{ No }} & \multicolumn{2}{|c|}{ TREN } & \multirow{3}{*}{$\begin{array}{c}\text { Total } \\
124 \\
\end{array}$} \\
\hline & & \multirow{2}{*}{$\begin{array}{c}\text { No } \\
14 \\
\end{array}$} & \multirow{2}{*}{$\begin{array}{c}\text { Sí } \\
110 \\
\end{array}$} & \\
\hline ¿Por qué motivo elige los & RAPIDEZ & & & \\
\hline medios de transporte que & ECONOMICO & 11 & 31 & 42 \\
\hline & $\begin{array}{l}\text { CERCANIA A PARADA O } \\
\text { ESTACION }\end{array}$ & 20 & 7 & 27 \\
\hline & COMODIDAD & 102 & 3 & 105 \\
\hline & SEGURIDAD & 2 & o & 2 \\
\hline \multicolumn{2}{|l|}{ Total } & 149 & 151 & 300 \\
\hline
\end{tabular}

Por último, es interesante destacar la diferencia que se observó entre los grupos en la variable vinculada a las "actividades realizadas usualmente durante el viaje", manifestándose mayores niveles de Bienestar Social en aquellos sujetos que realizan alguna actividad como "leer un libro" o "uso del celular (incluyendo redes sociales, radio, música)" por sobre aquellos que respondieron que no hacen ninguna actividad específica durante el recorrido.

Cuadro 8. Bienestar social y actividad realizada durante el viaje. Notas: a. Prueba de Kruskal-Wallis; $b$. Variable de agrupación: ¿Qué realiza USUALMENTE mientras viaja?. Fuente: elaboración propia en base al reporte de resultados del paquete estadístico SPSS 21.0.

\begin{tabular}{|c|c|c|}
\hline & $\begin{array}{c}\text { ¿Qué realiza USUALMENTE } \\
\text { mientras viaja? }\end{array}$ & Rango promedio \\
\hline \multirow[t]{6}{*}{ Integración Social } & USO DEL CELULAR & 146.99 \\
\hline & LEO UN LIBRO & 177.15 \\
\hline & LEO EL DIARIO & 162.55 \\
\hline & NO HAGO NADA & 112.39 \\
\hline & ESTUDIO & 181.00 \\
\hline & CONVERSO CON PERSONAS & 124.25 \\
\hline \multirow[t]{6}{*}{ Aceptación Social } & USO DEL CELULAR & 141.43 \\
\hline & LEO UN LIBRO & 198.70 \\
\hline & LEO EL DIARIO & 120.80 \\
\hline & NO HAGO NADA & 120.11 \\
\hline & ESTUDIO & 213.63 \\
\hline & CONVERSO CON PERSONAS & 169.75 \\
\hline \multirow[t]{6}{*}{ Contribución Social } & USO DEL CELULAR & 138.38 \\
\hline & LEO UN LIBRO & 202.06 \\
\hline & LEO EL DIARIO & 137.65 \\
\hline & NO HAGO NADA & 129.99 \\
\hline & ESTUDIO & 197.13 \\
\hline & CONVERSO CON PERSONAS & 136.25 \\
\hline
\end{tabular}




\begin{tabular}{|c|c|c|c|c|c|}
\hline \multirow{6}{*}{\multicolumn{2}{|c|}{ Actualización Social }} & \multirow{2}{*}{\multicolumn{2}{|c|}{ USO DEL CELULAR }} & \multicolumn{2}{|c|}{140.71} \\
\hline & & & & \multicolumn{2}{|c|}{190.85} \\
\hline & & \multicolumn{2}{|c|}{ LEO EL DIARIO } & \multicolumn{2}{|c|}{165.45} \\
\hline & & \multicolumn{2}{|c|}{ NO HAGO NADA } & \multicolumn{2}{|c|}{121.59} \\
\hline & & \multicolumn{2}{|c|}{ ESTUDIO } & \multicolumn{2}{|c|}{157.38} \\
\hline & & \multicolumn{2}{|c|}{ CONVERSO CON PERSONAS } & \multicolumn{2}{|c|}{156.17} \\
\hline \multirow{6}{*}{\multicolumn{2}{|c|}{ Coherencia Social }} & \multicolumn{2}{|c|}{ USO DEL CELULAR } & \multicolumn{2}{|c|}{146.92} \\
\hline & & \multicolumn{2}{|c|}{ LEO UN LIBRO } & \multicolumn{2}{|c|}{186.06} \\
\hline & & \multicolumn{2}{|c|}{ LEO EL DIARIO } & \multicolumn{2}{|c|}{117.00} \\
\hline & & \multicolumn{2}{|c|}{ NO HAGO NADA } & \multicolumn{2}{|c|}{124.49} \\
\hline & & \multicolumn{2}{|c|}{ ESTUDIO } & \multicolumn{2}{|c|}{130.13} \\
\hline & & \multicolumn{2}{|c|}{ CONVERSO CON PERSONAS } & \multicolumn{2}{|c|}{139.25} \\
\hline \multicolumn{6}{|c|}{ Estadísticos de contraste ${ }^{\mathrm{a}, \mathrm{b}}$} \\
\hline & $\begin{array}{l}\text { Integración } \\
\text { Social }\end{array}$ & $\begin{array}{l}\text { Aceptación } \\
\text { Social }\end{array}$ & $\begin{array}{l}\text { Contribución } \\
\text { Social }\end{array}$ & $\begin{array}{l}\text { Actualización } \\
\text { Social }\end{array}$ & $\begin{array}{l}\text { Coherencia } \\
\text { Social }\end{array}$ \\
\hline Chi-cuadrado & 15.931 & 27.525 & 26.117 & 19.332 & 15.219 \\
\hline $\mathrm{Gl}$ & 5 & 5 & 5 & 5 & 5 \\
\hline Sig. asintót. & .007 & .000 & .000 & .002 & .009 \\
\hline
\end{tabular}

\section{Discusión}

En el apartado introductorio a la temática abordada en la presente investigación, se argumentó acerca del interés de relacionar determinadas variables con el uso de los medios de transporte. Cabe destacar la importancia del uso de los medios para trasladarse, exponiendo las ideas de Gutiérrez (2009), quién hace referencia a una "práctica", en la que se reiteran conductas sociales en un determinado contexto. La mayoría de los usuarios del transporte, realizan un recorrido diario y rutinario, para llevar a cabo las actividades de su vida diaria.

El interés marcado en el transporte ferroviario se debe, a partir del presente estudio, tanto al recorrido histórico como al malestar que genera a nivel social por diversos motivos. A raíz de accidentes y desmejoras en los servicios, se genera un clima negativo tal, que los usuarios manifiestan su disconformidad (Rebón et al., 2010).

En los distintos transportes públicos, como el ferrocarril y los colectivos, al interactuar distintas personas con una misma finalidad, i.e., trasladarse de un lugar a otro, se generan emociones a nivel grupal, generando un clima emocional que dependerá de las emociones que se presenten a nivel colectivo.

El Bienestar Social es definido por Keyes (1998) como la apreciación que las personas hacen de las circunstancias y el funcionamiento dentro de la sociedad, tomando en consideración lo subjetivo y lo social, e influyendo directamente en el bienestar y la salud. El autor le da importancia al mundo que le es dado al sujeto y aquel que se construye intersubjetivamente.

Esta variable genera interés a la hora de analizar la influencia del transporte público en las personas a nivel social e individual, y se ve reflejada en los resultados obtenidos en el presente estudio; aquellos que utilizan el transporte ferroviario, presentan niveles significativamente bajos en las dimensiones del Bienestar Social. Dicho fenómeno, podría entenderse a partir del comportamiento de las dimensiones Aceptación e Integración, 
que tienen que ver con la confianza, aceptación y relaciones positivas con los demás, y por otra parte, la Contribución, Actualización y Coherencia Social, las que consideran cierta actividad del sujeto en la sociedad. Asimismo, es importante recordar que las personas que utilizan este medio de transporte, en general, se ubican en el primer y segundo quintil de ingresos per cápita, cuya posición económica y de clase social podría relacionarse con una visión social negativa, en tanto la precariedad de recursos, las importantes dificultades para satisfacer necesidades individuales, podrían repercutir sobre percepciones, sentimientos displacenteros o creencias de desesperanza, o sobre el bienestar social auto-percibido.

Este medio de transporte masivo, es utilizado por una parte de la sociedad para trasladarse diariamente, y del uso del mismo depende la llegada a tiempo para la realización de una actividad diaria, en el caso del ferrocarril el principal motivo es la ida y vuelta al empleo, u otro tipo de actividades comunes (e.g., la asistencia a alguna institución educativa de inicial, medio o superior, la llegada temprano a un turno médico), si se tiene en cuenta el mal funcionamiento del mismo y la calidad de viaje, se entienden los resultados obtenidos infiriendo que esta actividad diaria, i.e., el traslado de un lugar a otro, influye directamente en la percepción del Bienestar Social, tanto en sus dimensiones que se relacionan con la confianza y las actitudes positivas con los demás, como también con aquellas que contemplan la parte activa del sujeto en la sociedad como la Contribución y la Coherencia Social, sustantivamente. En relación a lo mencionado, Pírez (1999) sostiene que existe un desligamiento social y una incapacidad de decisión social frente a la desmejora del servicio de transporte férreo. Además, ante las situaciones de colapso por el mal funcionamiento del ferrocarril, se manifiesta una disconformidad en los usuarios (en consonancia con los argumentos de Rebón et al., 2010), y esto se ve reflejado en el presente estudio, en las bajas puntuaciones de Bienestar Social de los usuarios, contrastados con quienes eligen otros medios, ya que como explica Fuentes (2012), lo imprevisible del funcionamiento del tren, hace que las personas busquen alternativas de desplazamiento, incluyendo entre estas al transporte colectivo.

Por otro lado, los Valores Humanos son definidos por Schwartz (2001) como metas deseables, cambiantes, dependiendo de las situaciones eventuales y son útiles en la vida de las personas justificando las conductas. Cada cultura y grupo social tiene influencia sobre estas metas motivacionales.

En relación a la asociación entre los Valores Humanos y el Bienestar Social, estudios previos (Zubieta, Fernández y Sosa, 2012), han arrojado asociaciones positivas significativas de la mayoría de las dimensiones con los valores de Auto-trascendencia y Apertura al cambio. El presente estudio, también arrojó asociaciones positivas de todas las dimensiones del Bienestar Social con los valores de Autotrascendencia (i.e., valores de Universalismo y Benevolencia) y con el valor Seguridad; los primeros tienen que ver con una prevalencia de intereses colectivos, y el segundo con la armonía y estabilidad social. Otra relación importante a destacar, es la asociación negativa y significativa entre las dimensiones Aceptación, Contribución, Actualización y Coherencia Social con el valor Poder, entendiendo que el último valor mencionado tiene que ver con intereses personales de autoridad y poder social, predominando aspectos individualistas, mientras que las dimensiones del Bienestar Social mencionadas se relacionan en mayor medida con intereses relativos al óptimo funcionamiento social, considerando al sujeto como un agente o actor "activo" dentro de la sociedad, prevaleciendo la importancia del bien común y trascendiendo lo individual por lo social.

Al analizar la diferencia en los Valores Humanos que existe entre los usuarios de ferrocarril y aquellos que prefieren otros medios de transporte, se evidenciaron en los últimos mayores niveles de los Valores Benevolencia, Conformidad, Seguridad, los cuales tienen 
que ver con la armonía en las relaciones con los demás, la protección y el orden social (Fontaine et al., 2008), lo que podría entenderse en el sentido de que aquellas personas que utilizan otros medios distintos al ferrocarril contemplan intereses de mayor orden social, seguridad y protección. Por lo mismo, un alto porcentaje de usuarios de tren lo eligen por su rapidez dejando de lado la comodidad, mientras aquellos que eligen otros medios de desplazamiento, optan por la comodidad y por esto, como ya se mencionó, podrían poseer puntuaciones más elevadas en dichos valores.

En el medio de transporte automotor colectivo, existen otras variables como la espera, imprevisibilidad y el tránsito demorado (e.g., congestionamientos en accesos a la ciudad, manifestaciones o reclamos populares, etc.), pero muchos usuarios que optan por este medio pueden tener horarios de llegada más flexibles, o al tomarlo desde su terminal, prefieren viajar sentados dejando de lado la rapidez, los horarios programados y lo económico que caracteriza al transporte ferroviario. Particularmente, el desarrollo y modernización en el uso de la tecnología posibilita, para el modo de transporte ferroviario, la utilización de aplicaciones gratuitas -apps- para teléfonos celulares de tipo smartphones o bien para tablets con acceso a internet, permitiendo informar a los usuarios acerca del funcionamiento y pudiendo prever la llegada de las sucesivas formaciones a cada estación ferroviaria (e.g., Moovit); de modo más limitado, a través de este tipo de aplicaciones pueden preverse también las demoras en el medio colectivo en líneas que se desplazan por la CABA (i.e., líneas 32, 95, 124, 143, 152, entre otras). Con todo, es relevante legitimar el impacto actual de la tecnología sobre la situación de espera de cada sujeto y su impacto psicológico, lo mismo que otros aspectos infraestructurales del sistema de transporte público argentino, tales como la modernización del material rodante y eléctrico (i.e., adquisición de nuevas formaciones ferroviarias, instalación de acondicionadores de aire en gran parte de los trenes y ómnibus en funcionamiento).

En la misma línea, los valores de Logro, Hedonismo, Estimulación y Autodirección también presentaron niveles más elevados en aquellos individuos que no utilizan el ferrocarril como medio de transporte preferente. Dicha asociación podría entenderse a partir de una búsqueda del cumplimiento de los intereses individuales y del placer, considerando que apuntan al medio que menos malestar les genere para trasladarse, teniendo en cuenta el elevado porcentaje de usuarios de colectivos que lo eligen por comodidad.

Frente a los resultados obtenidos y desarrollados hasta aquí, podemos hacer referencia a estudios realizados en distintos momentos por Simmel (1903), Milgram (1970) y Miller (1964) (citados en Dorantes Rodríguez y Matus García, 2002), acerca del desequilibro psicológico que resulta de la vida en las ciudades, generando un filtro en la información que reciben las personas y evitando contactos sociales. Se podría entender por los mismos motivos a aquellos usuarios que prefieren viajar en condiciones más cómodas, sin dar mayor importancia a la rapidez. Pírez (1999) desarrolla acerca del desligamiento de la responsabilidad social que afecta a los usuarios de transporte y a la vida en las masas, agregando que existe una disminución del respeto en las relaciones interpersonales.

Otros resultados obtenidos que interesa destacar, refieren a la asociación de dichas variables con aquellos datos sociodemográficos que caracterizan al modo de viaje, que se consideraron importantes para la presente investigación. Se reportó una relación entre el horario de viaje y en el Bienestar Social, observándose una baja percepción de las dimensiones del Bienestar Social en aquellos usuarios que viajan de ida a la $\mathrm{CABA}$, antes de las 09:00 am, mientras que aquellos que viajan después de las 09:00 am tienen valores más elevados en las dimensiones Integración, Aceptación y Actualización Social, lo mismo ocurre en el caso del horario de antes de las 17:00 hs., de vuelta a GBA Sur. 
Para entender la información descripta arriba, es necesario considerar datos del Instituto del Transporte, de la Academia Nacional de Ingeniería (2011). Dichos datos sostienen que todas las líneas presentan un problema de saturación y de falta de comodidad en determinados rangos horarios, por lo cual entendemos que la "hora pico", es el momento del día en el que una mayor cantidad de personas se trasladan en un mismo momento y esto influye de manera directa en la percepción del funcionamiento social. Quienes viajan antes de las 09:00 am, poseen mayores limitaciones a la hora de pensar en el correcto funcionamiento social, evidenciándose en las bajas puntuaciones en la dimensión Actualización Social.

Por otra parte, se halló una interesante asociación entre la variable vinculada al tipo de desplazamiento en el medio de transporte (i.e., “iviajan sentados?”), en tanto se presentaron niveles más elevados en las dimensiones del Bienestar Social en aquellos que respondieron que "siempre", "a veces" o "frecuentemente" viajan sentados; lo cual se entiende, si se considera que viajar sentado es una condición de comodidad, pudiendo la misma influir en el Bienestar Social auto-percibido frente a cada situación cotidiana.

A modo de conclusión, podríamos considerar la relevancia multidisciplinar de la presente investigación, ya que los resultados obtenidos refuerzan hallazgos concluyentes de investigaciones de diversas disciplinas que estudian acerca de la influencia de los medios de transporte en la sociedad. El desplazamiento es un hecho social e influye de manera directa en las actividades cotidianas de las personas, por lo cual es necesario que se desnaturalicen las malas condiciones de viaje.

Conocer las variaciones del Bienestar Social en usuarios de transporte público y la relación de sus dimensiones con los Valores Humanos, abre un amplio abanico de intereses hacia disciplinas varias, ya que repercute en el interés de quienes estudian el funcionamiento social, la aglomeración de las grandes ciudades y las condiciones de los medios de transporte.

Contemplando los resultados desarrollados en la presente investigación, llevada a cabo entre los años 2015 y 2016, podrían considerarse las características del tipo de traslado que los usuarios eligen, como una posible causa de malestar intersubjetivo, nutriendo la temática de la movilidad y el transporte, considerando bajo este panorama posibles mejoras futuras de los servicios con la finalidad de mejorar la calidad de viaje de los usuarios.

En línea con lo anterior, algunos autores (e.g., Cresswell, 2010; Sheller y Urry, 2006; Urry, 2004, 2007) observan que las agendas contemporáneas de investigación en materia de realización de estudios vinculados al transporte no contemplan el significado holístico de la movilidad, entendida como una cuestión paradigmática definida por el movimiento físico cotidiano imperante en las sociedades sobre todo a partir del Siglo $\mathrm{XX}$, i.e., caminar, moverse a través de bicicletas, autobuses, automóviles, trenes, barcos, aviones u otro tipo de tecnologías modernas, debiendo examinar la manera en que el transporte de personas y la comunicación de mensajes e imágenes de modo local, internacional y/o globalizado (i.e., digitalización a través de internet) influyen individual o colectivamente sobre una sociedad o cultura específica y las interdependencias entre diversos agentes y usuarios.

Las transformaciones y reestructuraciones del ferrocarril argentino durante la década del 90, dadas en el marco de un proceso de privatización ferroviaria, que si bien dieron como resultado la reducción del servicio de pasajeros hacia algunos circuitos turísticos y hacia la provincia de Buenos Aires, el incremento del número de estaciones y ramales, y la densidad de la red para el AMBA expresada en una mayor cantidad de pasajeros urbanos, mejoras en la seguridad e higiene de los coches, y un aumento tarifario, significó la caída 
de un "servicio social" en el interior del país (Benedetti, 2016:82), obligando a las provincias a transferir el tráfico a la modalidad de ómnibus interurbano. Bajo este panorama, el cambio radical de las políticas ferroviarias argentinas acaecidas hacia fines del Siglo XX, ha influido en la organización territorial regionalizando el transporte, repercutiendo sobre las formas de movilidad de las personas, los intercambios dados entre el interior del país y la capital e, incluso, mostrando actualmente diferencias en los pasajeros provenientes de las áreas urbanas. Cabe pensar que tales procesos reorganizativos del transporte, y las actuales modernizaciones del sistema, tendrían un efecto sobre el bienestar psicológico y el clima socioemocional de los usuarios; en tanto, los cambios dinámicos dados en las últimas décadas sobre el sistema ferroviario y, en general, sobre las políticas de transporte público, instarían a los usuarios a modificar sus esquemas, creencias y visiones acerca de los medios en los que se movilizan cotidianamente.

Esta mirada psicosocial del transporte se emparenta con el paradigma de la movilidad esbozado en el párrafo anterior, en cuyo escenario se entraman movimientos y afectos que difieren conforme al tipo de transporte utilizado habitualmente (e.g., Löfgren, 2015). Singh (2016) analiza culturalmente las prácticas de movilidad definiendo los espacios en las que éstas se manifiestan, i.e., el transporte público, como "espacios sociales", históricamente reconstruidos, donde emergen relaciones entre pasajeros, creencias individuales o representaciones sociales, comportamientos de autodisciplina social y proximidad corporal, donde factores psicosociales como el Bienestar Social y los Valores Humanos podrían tener activa participación. Considerando los hallazgos del presente estudio y el contexto socio-histórico de cambios que ha experimentado el sistema de transporte público en los últimos años, los Valores Humanos de Autotrascendencia, i.e., la primacía del colectivismo sobre el desarrollo y bienestar individual, legitiman la consideración antropológica del constructo relacionado a la movilidad. Al respecto, en palabras de Singh (2016:23),

(...) la movilidad urbana puede ser abordada desde la experiencia en tanto fenómeno social y cultural, lo que permite conocer las prácticas, representaciones e interacciones de los pasajeros durante el viaje como un modo más de habitar la ciudad, complementando aspectos ya abordados en los estudios de transporte -como son la accesibilidad, uso del tiempo, desigualdad-y ampliando el conocimiento sobre los usos del espacio en los estudios urbanos. En segundo lugar, la perspectiva histórica puede ayudarnos a comprender cómo las prácticas cotidianas de viajar en la ciudad se construyen, reproducen y cambian a lo largo del tiempo.

Con todo, la presente investigación, aporta datos empíricos que podrían profundizarse en investigaciones futuras considerando algunos aspectos antropológicos del paradigma mencionado anteriormente, y teniendo en cuenta principalmente la mirada psicosocial, hacia las conductas de las personas en sociedad, con el fin de construir estrategias de cambio o mejora en el bienestar a nivel colectivo, y en la calidad de las relaciones interpersonales en el marco de propiciar el desarrollo genuino de políticas de movilidad sobre las cuales se regulen los sistemas públicos de transporte, a fin de asegurar que la ciudadanía logre alcanzar óptimamente, y experimentando los mínimos niveles de estrés posible, sus necesidades y objetivos cotidianos (i.e., rutinas laborales, académicas, familiares, sanitarias, etc.). 


\section{Q Bibliografía}

» BABASE, Nekane (2004) Salud, Factores Psicosociales y Cultura. En D. Páez, I. Fernández, S. Ubillos, \& E. Zubieta (Coords.). Psicología social, cultura y educación, pp. 891-913. Madrid: Pearson Prentice Hall.

" BENEDETTI, Alejandro (2016) Argentina, ¿país sin ferrocarril? La dimensión territorial del proceso de reestructuración del servicio ferroviario $(1957,1980$ y 1998). Revista Transporte y Territorio, 15, 68-85.

» BILBAO, María de los Ángeles, TECHIO, Elza y PÁEZ, Darío (2007) Felicidad, Cultura y valores personales: Estado de la cuestión y síntesis meta-analítica. Revista de Psicología, 25(2), 233-276.

" BLANCO, Amalio y DíAZ, Darío (2005) El bienestar social: su concepto y medición. Psicothema, 17(4), 582-589.

" BLANCO, Amalio y DíAZ, Darío (2006) Orden social y salud mental: una aproximación desde el bienestar social. Revista Clínica y Salud, 17, 7-29.

»CASTRO SOLANO, Alejandro (2005) Técnicas de evaluación psicológica en los ámbitos militares. Buenos Aires: Paidós.

» CRESSWELL, Tim (2010) Towards a politics of mobility. Environment and Planning D: Society and Space, 28, 17-31. doi: 10.1068/d11407

»DORANTES RODRÍGUEZ, Carlos Héctor y MATUS GARCÍA, Graciela (2002) El estrés y la ciudad. Revista del Centro de Investigación, 18(5), 71-77.

» FONTAINE, Johnny R. J., POORTINGA, Ype H., DELBEKE, Luc. y SCHWARTZ, Shalom H. (2008) Structural equivalence of the values domain across cultures. Distinguishing sampling fluctuations from meaningful variation. Journal of Cross-Cultural Psychology, 39(4), 345-465.

» FUENTES, Ariel Rodolfo (2012) Cuestiones urbanas y regionales en Argentina. Procesos y prácticas en la ciudad contemporánea. Territorios en construcción. Ponencia presentada en VII Jornada de Sociología de la UNLP. Universidad Nacional de La Plata, La Plata, Argentina.

» GUTIÉRREZ, Andrea (2009) Movilidad o inmovilidad: ¿Qué es la movilidad? Aprendiendo a delimitar los deseos. XV Congreso Latinoamericana de Transporte Público y Urbano, XV CLATPU, Buenos Aires.

» INSTITUTO DEL TRANSPORTE ACADEMIA NACIONAL DE INGENIERÍA. (2011) Accesos a la región metropolitana de Buenos Aires. El transporte ferroviario y los subterráneos. Recuperado de http://www.acadning.org.ar/Institutos/ ANI\%20-instituto\%2odel\%20Transporte\%20Estudio\%20Estrategico\%20 Preliminar\%20Accesos\%20RM\%20\%20\%20vo03.pdf

" INTERNATIONAL BUSINESS MACHINES Corporation $(1989,2012)$ Manual del usuario del sistema básico de IBM SPSS Statistics 21. Chicago, ILL: SPSS Inc.

» KEYES, Corel (1998) Social well-being. Social Psychology Quarterly, 61, 121-140.

» KEYES, Corel, RYFF, Carol y SHMOTKIN, Dov (2002) Optimizing well-being: the empirical encounter of two traditions. Journal of Personality and Social Psychology, 82, 1007-1022. 
" LÖFGREN, Orvar (2015) Modes and Moods of Mobility: Tourists and Commuters. Culture Unbound: Journal of Current Cultural Research, 7, 175-195.

" MARTíNEZ, Fernando, DE CRISTÓFARO, Gilda, SÁNCHEZ, Laura, HANTKE, Benjamín y DíAZ, Alejandro (2014) "El sistema de transporte en Argentina" Diagnóstico y propuestas para su Desarrollo. (CECREDA informe). Recuperado del sitio de internet del Centro Estratégico para el Crecimiento y Desarrollo Argentino: http://www.cecreda.org.ar/archivos/nNiKb.pdf

» MINISTERIO DE TRANSPORTE, Comisión Nacional de Regulación del Transporte (2015) Informe estadístico 2015 de la red ferroviaria argentina. Recuperado de http://www.cnrt.gob.ar/content/estadisticas/ferroviario

» MüLLER, Alberto (2015) Ante un nuevo ciclo: delineando un futuro para el ferrocarril interurbano en la Argentina. (Documento $n^{\circ} 42$ ). Recuperado de http://blogdelcespa.blogspot.com.ar/p/documentos-de-trabajo.html

» PÁEZ, Darío y ZUBIETA, Elena (2006) Dimensiones Culturales. IndividualismoColectivismo como Síndrome Cultural. En: D. Paez, I. Fernandez, S. Ubillos \& E. Zubieta. Psicología Social, Cultura y Educación. Madrid: Pearson Educación (pp.56-87)

» PÉREZ, Verónica y REBÓN, Julián (2017) La perturbación como motor de la historia. Los ferrocarriles metropolitanos durante el kirchnerismo. Ciudad Autónoma de Buenos Aires: Biblos.

» PÉREZ, Verónica (2010) La gestión del sistema ferroviario urbano de pasajeros. Un terreno fértil para el desborde hostil de los disconformes (1994-2008). Revista Transporte y Territorio, 3, 103-123.

" PíREZ, Pedro (1999) Gestión de servicios y calidad urbana en la ciudad de Buenos Aires. EURE Revista Latinoamericana de Estudios Urbano Regionales, 25(76), 125-139.

» PRESIDENCIA DE LA NACIÓN (2009-2010) Encuesta de Movilidad Domiciliaria. Buenos Aires: Ministerio de Transporte. Recuperado del sitio de internet del Ministerio de Transporte de la Nación http://uecmovilidad.gob.ar/encuestade-movilidad-domiciliaria-2009-2010-movilidad-en-el-area-metropolitana-debuenos-aires/

» REBÓN, Julián y HERNÁNDEZ, Candela (2017) Las formas de la acción colectiva en el sistema ferroviario de pasajeros de la Región Metropolitana de Buenos Aires. Revista Transporte y Territorio, 16, 201-219.

» REBÓN, Julián, QUINTANA, Gustavo, PÉREZ, Verónica, ÁLVAREZ, Jorge, GAMALLO, Leandro, HERNÁNDEZ, Mariela Julia, y TAFURO, Sebastián (2010) Trenes en Ilamas. La disconformidad social entre los usuarios del sistema público de transporte ferroviario del área metropolitana de Buenos Aires. Revista Theomai, 21, 77-95.

» SCHWARTZ, Shalom (1992) Universals in the content and structure of values: Theoretical advances and empirical test in 20 countries. New York: Academic Press.

»SCHWARTZ, Shalom (2001) ¿Existen aspectos universales en la estructura y contenido de los valores humanos? En: M. Ros y V. Gouveia (Eds.), Psicología Social de los valores humanos (pp. 53-76). Madrid: Biblioteca Nueva.

» SCHWARTZ, Shalom, MELECH, Gila, LEHMAN, Arielle, BURGESS, Steven, HARRIS, Mari y OWENS, Vicki (2001) Extending the cross-cultural validity of the theory of basic human values with a different method of measurement. Journal of Cross Cultural Psychology, 32(5), 519-542. 
»SHELLER, Mimi y URRY, John (2006). The new mobilities paradigm. Environment and Planning A, 38, 207-226. doi: 10.1068/a37268

"SINGH, Dhan Zunino (2016) "Sea amable, ceda el asiento". Un análisis histórico cultural del comportamiento de los pasajeros en el transporte público de Buenos Aires a principios del siglo XX. Cuaderno Urbano. Espacio, Cultura, Sociedad, 20(20), 5-25.

"SZENKMAN, Paula (2015) Menos autos y mejor transporte público para la Región Metropolitana de Buenos Aires. (CIPPEC análisis 149). Recuperado del sitio de internet de Centro de Implementación de Políticas Públicas para la Equidad y el Crecimiento: Recuperado de http://www.cippec.org/documents/ 10179/51825/149+DPP+ADE,\%20Menos+autos+y+m\%C3\%A1s+y+mejor+tran sporte + p\% $3 \%$ BAblico+para+la+Regi\%C3\%B3n+Metropolitana+de+Bueno s+Aires, \%20Szenkman+2015.pdf/afbo97fb-5f95-4301-bc2e-9f95of118a30

» URRY, John (2004) The system of automobility. Theory, Culture and Society 21(4/5), 25-39.

»URRY, John (2007) Mobilities. Cambridge: Polity Press.

"ZUBIETA, Elena, DELFINO, Gisela y FERNÁNDEZ, Omar (2007) Confianza institucional y el rol mediador de creencias y valores. Revista de Psicología, 3(6) 101-120.

» ZUBIETA, Elena, FERNÁNDEZ, Omar y SOSA, Fernanda (2012) Bienestar, valores y variables asociadas. Boletín de Psicología, 106, 7-27.

\section{María Luciana Della Pittima / mluciana.dp@gmail.com}

Licenciada en Psicología de la Universidad Abierta Interamericana (UAI), con filiación institucional en la Facultad de Psicología y Relaciones Humanas de la UAI.

\section{Pablo Domingo Depaula / lic_pablodepaula@yahoo.com.ar}

Doctor en Psicología de la Universidad de Buenos Aires (UBA), Magister en Psicología Organizacional con Orientación Gerencial de la Universidad Abierta Interamericana (UAI), y Licenciado en Psicología de la Universidad Abierta Interamericana (UAI), con filiación institucional en el Consejo Nacional de Investigaciones Científicas y Técnicas (CONICET), Instituto de Investigaciones en Psicología de la UBA. 